\title{
Identification of biomechanical nonlinearity in whole-body vibration using a reverse path multi-input-single-output method
}

\author{
Ya Huang $^{1}$, Neil Ferguson ${ }^{2}$ \\ ${ }^{1}$ School of Engineering, University of Portsmouth, UK \\ ${ }^{2}$ Institute of Sound and Vibration Research, University of Southampton, UK
}

Correspondence address:

Dr Ya Huang

School of Engineering,

University of Portsmouth

Portsmouth PO1 3DJ

England

Telephone: (+44) 02392842343

Facsimile: (+44) 02392842351

E-mail: Ya.Huang@port.ac.uk 


\section{Abstract}

The study implements a classic signal analysis technique, typically applied to structural dynamics, to examine the nonlinear characteristics seen in the apparent mass of a recumbent person during wholebody horizontal random vibration. The nonlinearity in the present context refers to the amount of 'output' that is not correlated or coherent to the 'input', usually indicated by values of the coherence function that are less than unity. The analysis is based on the longitudinal horizontal inline and vertical cross-axis apparent mass of twelve human subjects exposed to $0.25-20 \mathrm{~Hz}$ random acceleration vibration at 0.125 and $1.0 \mathrm{~ms}^{-2}$ r.m.s. The conditioned reverse path frequency response functions (FRF) reveal that the uncorrelated 'linear' relationship between physical input (acceleration) and outputs (inline and cross-axis forces) has much greater variation around the primary resonance frequency between 0.5 and $5 \mathrm{~Hz}$. By reversing the input and outputs of the physical system, it is possible to assemble additional mathematical inputs from the physical output forces and mathematical constructs (e.g. square root of inline force). Depending on the specific construct, this can improve the summed multiple coherence at frequencies where the response magnitude is low. In the present case this is between 6 and $20 \mathrm{~Hz}$. The statistical measures of the response force time histories of each of the twelve subjects indicate that there are potential anatomical 'end-stops' for the sprung mass in the inline axis. No previous study has applied this reverse path multi-input-single-output approach to human vibration kinematic and kinetic data before. The implementation demonstrated in the present study will allow new and existing data to be examined using this different analytical tool.

Keywords: Whole-body vibration; biomechanics; nonlinear dynamics; multi-input-single-output 
The study applied a system identification procedure to analyse 'paths' that contributed to nonlinear dynamic behaviour of the human body during whole-body vibration (WBV). The nonlinearity in this context refers to the amount of 'output' response that is not linearly correlated to the 'input' excitation, usually indicated by values of the coherence function that are less than unity. This mathematical nonlinearity may be associated with the biomechanical nonlinearity, previously referred to as 'biodynamic nonlinearity', in which the resonance frequency increased with decreasing vibration magnitude [1,2]. Improved understanding of the mathematical nonlinearity may help to quantify the biomechanical nonlinearity that holds the key to dynamic response predictions at substantially different magnitudes.

Biomechanical nonlinearity has been reported in both the vertical and the fore-and-aft responses of the seated human body during vertical whole-body vibration [3], in both the fore-and-aft and vertical responses of the seated human body during fore-and-aft whole-body vibration [4,5], in both vertical and longitudinal horizontal responses of the recumbent person during vertical whole-body vibration [6], and in both longitudinal horizontal and vertical responses of the recumbent person during longitudinal horizontal whole-body vibration (see Figure 1). With recumbent subjects, any voluntary or involuntary movement and muscular activity were assumed to be reduced compared to a seated person. Therefore, it provided a better condition to examine the 'linearity' of a dynamic system in comparison with other postures [2].

By measuring dual axial responses of seated subjects exposed to simultaneous dual axial excitation, Mansfield and Maeda [7] observed similar magnitude dependency of the resonance frequency. The additional axis of excitation had a similar effect of increasing the vibration magnitude in a single axis excitation, with a reduced resonance frequency characteristic in its apparent mass. Experimental studies like this would benefit from an analytical framework that could isolate effects of each axis of excitation on each axis of the response.

The intended procedure applied in this paper is called the reverse path nonlinear multi-input-singleoutput (MISO) method. It was introduced by Bendat et al. [8] and later demonstrated with implementations by Bendat and Piersol $[9,10]$. There are two principle steps: first, one needs to define and prepare 'mathematical' inputs, usually physical output from measurement of the structural response, and 'mathematical' output, usually the physical input excitation, in the reverse path diagram shown in Figure 2. Secondly, one produces a formulation of the MISO system including computation of frequency response functions (FRF) based on correlated and uncorrelated (or conditioned) mathematical inputs and their coherence functions (Figure 3). 


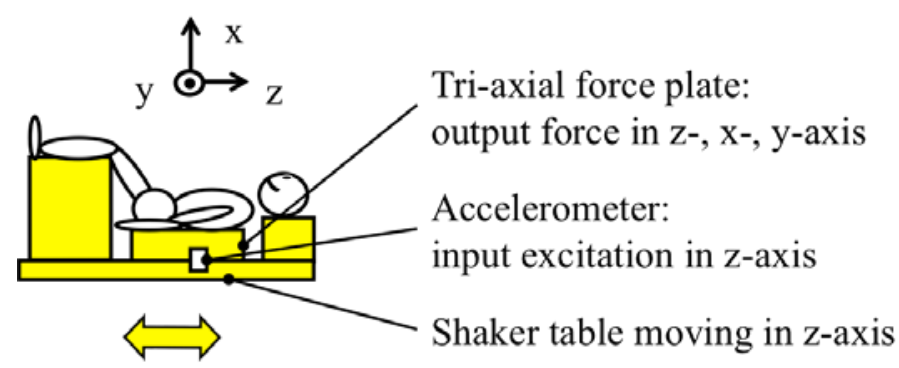

Figure $1 \quad$ Schematic experimental setup of the supine human body exposed to horizontal vibration and the axes of the forces (in horizontal z-axis, vertical $x$-axis and lateral y-axis) and acceleration (z-axis) transducers following Huang and Griffin [1].

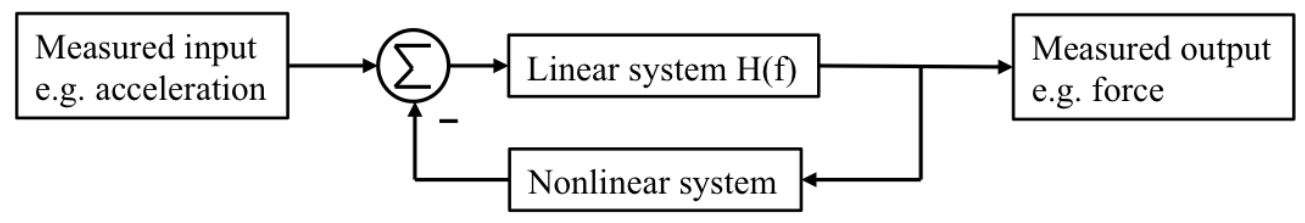

(a) Forward path diagram with feedback

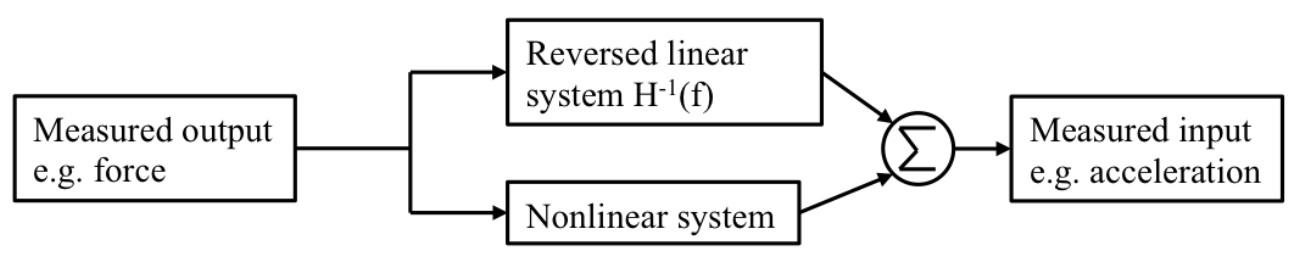

(b) Reverse path diagram without feedback

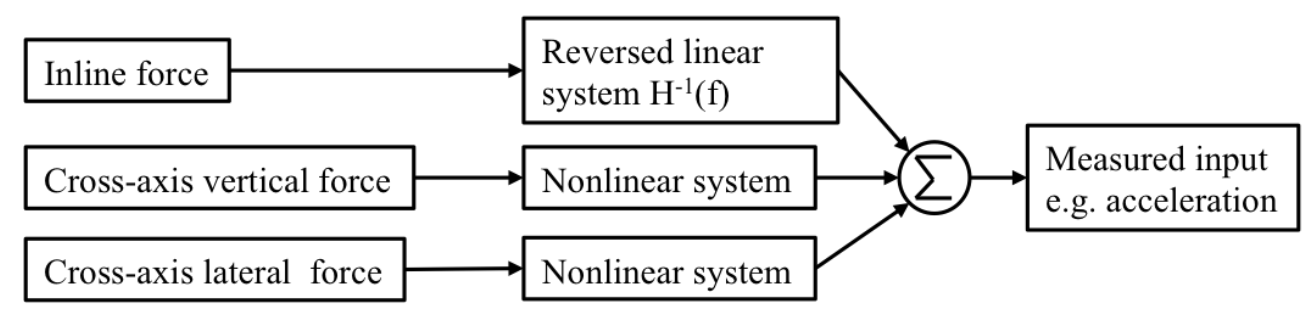

(c) Specified reverse path diagram without feedback

Figure 2 Reverse path diagram (b) derived from the original forward path diagram (a) eliminates the feedback loop - a cumbersome computational procedure. Diagram (c) presents the reverse path MISO system implemented for the present study adapted from Bendat and Piersol [9].

The procedure has been widely used in structural dynamics to identify nonlinear behaviour present in flexible and slender structures. An example being the nonlinear cubic stiffening effect of a two-endclamped mid-excited beam, characterised by two nonlinear mathematical inputs in addition to the original dynamic force input [11]. The two added inputs were the square and cubed power of the input 
dynamic force. With the two mathematically constructed inputs, the multiple coherence function was markedly improved, enabling a more accurate prediction model to be used for the structural response when the ordinary coherence was low.

For road-induced vehicle vibration a MISO system was employed to analyse transmissibilities of multiple acceleration inputs [12]. Instead of using arbitrarily constructed mathematical inputs, the authors used multiple channels of physical inputs - up to twelve accelerations at the four corners of the seat floor and in each of the three orthogonal directions. The method identified the dominant channels for the input acceleration in predicting the seat transmissibility.

With longitudinal horizontal random excitation of a semi-supine human body, the coherence function of the apparent mass showed a drop between 6 and $20 \mathrm{~Hz}$ [1]. With increasing magnitude of excitation, the frequency of the coherence drop decreased - a similar behaviour to the resonance frequency of the apparent mass between 2 and $4 \mathrm{~Hz}$. It was plausible to assume that at certain frequencies a part of the output force in the inline (longitudinal) direction was transferred to the crossaxis (vertical) direction, and therefore the coherence of the inline apparent mass was low at these frequencies. However, there has been no investigation to quantify the amount of 'transferred' output force from the inline axis to the cross axis.

Most biomechanical studies of whole-body vibration have allocated any nonlinear effects at the 'output' side of a transfer function, e.g. [13]. From a system linearity point of view, it was not known whether the output could have a nonlinear feedback path to affect the linear input such as that shown in Figure 2a. At the same time, implementing a feedback loop in the frequency response functions (FRF) involves time-consuming iterative procedures and stringent assumptions about the random distribution of the output. A 'reversed path' approach would offer a more efficient computational algorithm for FRFs and coherences [9].

When using correlated inputs it is difficult to obtain separately the linear FRF, in an optimum leastsquares predicted sense, between the individual inputs and the output(s). The identification method applied in the present study enables firstly a primary input to be used to estimate the first linear transfer function between itself and the output. The correlated parts of the other inputs are identified separately and are expressed in terms of linear transfer functions between the primary input and the other inputs. A calculation is then made to estimate any further linear transfer function between the uncorrelated parts of the other inputs and the output. To obtain the optimum linear transfer functions for the other inputs, there is the iterative relationship relating the subsequent transfer functions between the uncorrelated components in addition to the contribution from the correlated components. In this manner the optimum linear transfer functions between each of the inputs can be evaluated. Bendat and Piersol [9] applied this procedure to the 'Duffing' oscillator model excited by a force. The method recovered the two linear transfer functions between the two 'inputs', namely the displacement 
and the cube of the displacement, and the applied force. The former transfer function incorporated the linear mass, stiffness and damping terms and the second transfer function estimated the constant coefficient multiplying the cubed displacement for example.

The present study examines the longitudinal inline and vertical cross-axis apparent mass of a supine body exposed to $0.25-20 \mathrm{~Hz}$ random vibration at 0.125 and $1.0 \mathrm{~ms}^{-2}$ r.m.s. acceleration measured by Huang and Griffin [1] (Figure 1). The original system of input excitation and output force measured at the driving point, i.e. the subject-support interface, was transformed into the reverse path diagram in Figure 2b. The longitudinal inline $\mathrm{z}$-axis force forms the first mathematical input, the vertical cross $\mathrm{x}$ axis force forms the second mathematical input, and the third mathematical input is arbitrary and could be formulated by either the lateral cross y-axis force, the square of the inline $\mathrm{z}$-axis force, or the square root of the inline z-axis force. The displacement-force relationship at the base could then be interpreted, for example, by a 'softening' stiffness component when a squared force construct is applied, as the third mathematical input, or a 'hardening' stiffness when a sign-conserved square rooted force construct is employed. The longitudinal z-axis excitation acceleration forms the mathematical output (Figure 2c). The purpose of the adapted reversed MISO scheme (reMISO) would be to obtain the linear frequency response functions after removing the effects from each mathematical input (or physical output). The study tried to demonstrate the effects of mathematically constructed inputs, e.g. the sign-conserved square root of the inline z-axis force, to the nonlinear relationship between physical input and physical output of a system. Such relationships will be examined using ordinary, partial and multiple coherences. No previous study has applied this reverse path multi-inputsingle-output approach to human vibration kinematic and kinetic data before. The implementation demonstrated in the present study will allow new and existing data to be examined using this different analytical tool.

\section{$2 \quad$ Method}

The present study focuses on the key variables used to implement the reversed multi-input-singleoutput (reMISO) method to examine the transfer function defined by apparent mass. Apparent mass is defined as the ratio between the resultant 'physical output' force measured at the driving point, i.e. the excitation-subject interface, and the excitation 'input' acceleration at the supporting surface or base. Extensive procedures to derive uncorrelated, or conditioned, mathematical inputs, correlated and uncorrelated FRF, ordinary, partial and multiple coherence functions have been demonstrated by Bendat and Piersol $[9,10]$. They were implemented with single physical input and single physical output by Sweitzer [11], with multiple physical inputs and outputs by Qiu and Griffin [12], and with both single input and multiple inputs by Mandapuram et al. [14]. 
The apparent masses of twelve semi-supine subjects measured at two magnitudes of continuous broadband random $(0.25$ to $20 \mathrm{~Hz})$ vibration, i.e. 0.125 and $1.0 \mathrm{~ms}^{-2}$ r.m.s., were examined using time histories from Huang and Griffin [1]. MATLAB 7.10 was used to perform all computational analysis.

The three mathematical inputs were defined from Figure 2 as:

$x_{1}$ - measured longitudinal inline response force at the driving point $(\mathrm{N})$.

$x_{2}$ - measured vertical cross-axis response force at the driving point $(\mathrm{N})$.

$x_{3}$ - constructed sign-conserved square root of the horizontal longitudinal $z$-axis inline response force at the driving point $\left(\mathrm{N}^{1 / 2}\right)$. So $x_{3}$ has the same sign and therefore phase as the $z$-axis inline force - it always produces a restoring force. The mathematical construct of the input is not unique. The choice of a square root function is purely arbitrary and the intention was to show a nonlinear dependency of the displacement or equivalently acceleration as a function of the force amplitude. So a displacement or acceleration proportional to the square root of the force would not increase to as large a value as a linear relationship at high enough force amplitudes. In contrast, a relationship proportional to the squared function of force would have the displacement and acceleration amplitude increasing nonlinearly at a larger rate than a linear relationship between them and the force. See the discussions in Section 4.1 where the interpretation is further elaborated, dependent upon the level of the force, acceleration and corresponding displacement.

The one mathematical output was defined from Figure 2 as:

$$
y \text { - measured longitudinal excitation acceleration at the base }\left(\mathrm{m} / \mathrm{s}^{2}\right) \text {. }
$$

In general, ' $H$ ' is used to denote transfer functions based on correlated original mathematical inputs, while ' $L$ ' denotes the transfer functions based on conditioned or uncorrelated mathematical inputs where correlated portions are removed from each path. The general algorithm to formulate the MISO system in operational order is provided in Appendix A. The present study was based on three inputs and one output. A simplified notation is summarised below with a schematic representation shown in Figure 3. 


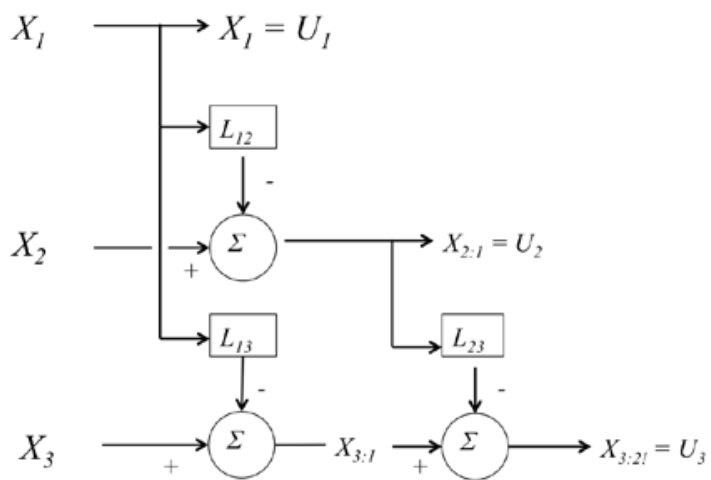

(a)

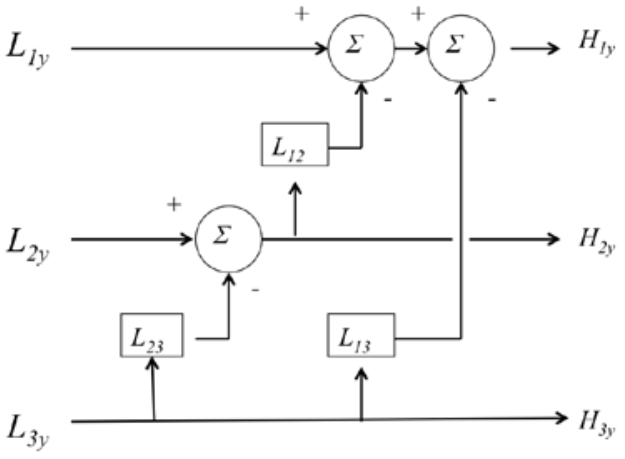

(b)

Figure 3 (a) Conditioned or uncorrelated mathematical inputs $\left(U_{1}, U_{2}, U_{3}\right)$ determined by recursive operation from correlated inputs $\left(x_{1}, x_{2}, x_{3}\right)$. (b) FRF of correlated inputs ' $H$ ' found from recursive sum of uncorrelated FRFs ' $L$ ' adapted from Bendat and Piersol [9].

The standard transfer function of (ordinary) apparent mass using cross spectral density (CSD) method takes the form:

$$
\begin{gathered}
H_{1}(f)=\frac{G_{o i}(f)}{G_{i i}(f)} \\
H_{2}(f)=\frac{G_{o o}(f)}{G_{i o}(f)} \\
H_{v}(f)=\frac{G_{o i}(f)}{\left|G_{o i}(f)\right|} \cdot\left(\frac{G_{o o}(f)}{G_{i i}(f)}\right)^{I / 2}
\end{gathered}
$$

and for the ordinary coherence function: $\operatorname{coh}_{i o}(f)=\frac{\left|G_{o i}(f)\right|^{2}}{G_{i i}(f) G_{o o}(f)}=\frac{H_{1}(f)}{H_{2}(f)}$

where, $f$ is frequency scale, $H_{1}(f)$ and $H_{2}(f)$ both measure the amount of the output that is linearly correlated to the input; $H_{1}(f)$ assumes that nonlinearity or noise comes from the output; $H_{2}(f)$ assumes that nonlinearity or noise comes from the input; $H_{v}(f)$ assumes that nonlinearity or noise comes from both the input and the output [15]. With normal single input multiple output, $H_{v}$ reduces to an equivalent of the 'PSD' estimator, i.e. $\left(G_{o o}(f) / G_{i i}(f)\right)^{1 / 2}$, but with the phase of $G_{o i}(f)$. The modulus of $H_{v}$ usually falls between those of the $H_{1}$ and $H_{2}$ estimators. $G_{o i}(f)$ is the cross spectral density (CSD) function between the physical output and input; $G_{i i}(f)$ and $G_{o o}(f)$ are the power spectral density (PSD) function for the physical input and output, e.g. $G_{11}$ is the PSD of the z-axis inline force as the first mathematical input; $\operatorname{coh}_{i o}$ is the (ordinary) coherence function between the physical input and output. In a normal sense, the input for the apparent mass is the acceleration excitation, and the output is the driving point dynamic force. However, in the reverse path method illustrated, the mathematical inputs 
will be constructed from the driving point dynamic force, i.e. $x_{1}, x_{2}$, and $x_{3}$, and the mathematical output will be the excitation acceleration, i.e. $y$.

The CSDs and PSDs were estimated via Welch's method at frequencies between 0.25 and $20 \mathrm{~Hz}$ with data shown between 0.25 and $10 \mathrm{~Hz}$. Each time history of $90 \mathrm{~s}$ was processed using a Fast Fourier transform (FFT) window length of 2048 samples, a Hamming window with 50\% overlap, a sampling rate of 200 samples per second and an ensuing frequency resolution of approximately $0.1 \mathrm{~Hz}$ [1].

The FRFs based on correlated mathematical inputs i.e. $H_{3 y}, H_{2 y}$, and $H_{1 y}$ take the form:

$$
\begin{aligned}
& H_{3 y}=L_{3 y} \quad \text { where } L_{3 y}=\frac{G_{3 y-2 !}}{G_{33-2 !}} \\
& H_{2 y}=L_{2 y}-L_{23} H_{3 y} \\
& H_{1 y}=L_{1 y}-L_{12} H_{2 y}-L_{13} H_{3 y}
\end{aligned}
$$

Subscripts 1, 2, 3 and $y$ refer to the three mathematical inputs and one output respectively, while '!' refers to the removal of all lower counts channels, e.g. '2!' means both $x_{1}$ and $x_{2}$ are removed from the function.

The FRFs based on uncorrelated conditioned mathematical inputs, i.e. $L_{2 y}$ and $L_{1 y}$, are:

$$
L_{l y}=\frac{G_{1 y}}{G_{11}} \quad L_{2 y}=\frac{G_{2 y-1}}{G_{22 I}}
$$

The ordinary coherence functions for the conditioned inputs are:

$$
\begin{aligned}
& \operatorname{coh}_{u l y}=\frac{G_{y I} G_{l y}}{G_{I I} G_{y y}} \quad \text { which is the same as the ordinary coherence function } \\
& \operatorname{coh}_{u 2 y}=\frac{G_{y 2 I I} G_{2 y-I}}{G_{22-I} G_{y y}} \quad \operatorname{coh}_{u 3 y}=\frac{G_{y 3-2 !} G_{3 y-2 !}}{G_{33-2 !} G_{y y}}
\end{aligned}
$$

where subscript $u$ denotes uncorrelated inputs, e.g. $u_{1}=G_{11}, u_{2}=G_{22.1}, u_{3}=G_{33.2 !}$, see Appendix A for $G_{j j . r !}$.

The multiple coherence function as a summation of all uncorrelated contribution of inputs is:

$$
\operatorname{coh}_{y x}=\operatorname{coh}_{u l y}+\operatorname{coh}_{u 2 y}+\operatorname{coh}_{u 3 y}
$$

The algorithm used above computes the ordinary coherence functions of conditioned inputs as a percentage of the uncorrelated input $u$ to the total output $\left(G_{y y}\right)$ and then sum them up [9]. These are used in the present study, as the ordinary coherence functions of conditioned inputs offer more physically meaningful interpretation of each uncorrelated input with regard to the overall output. 
The linear FRF determined from the mathematical output $y$ and the original mathematical input $x_{1}$ is then expressed as:

$$
H_{y l}=\frac{l}{H_{l y}}
$$

A linear two-pole-two-zero fit of the $H_{y 1}$ function was then performed so as to estimate its peak frequency. Peak frequencies for the ordinary FRF $H_{1}$ were previously obtained by fitting a parallel two-degree-of-freedom lumped parameter mass-spring-damper system in the frequency range 0 to 10 $\mathrm{Hz}$ by Huang and Griffin [1].

The peak frequencies extracted from the ordinary and conditioned FRF of the twelve subjects at the two vibration magnitudes are tested using the Wilcoxon's matched-pairs signed ranks test. This class of statistical methods was 'distribution-free' or 'non-parametric'. They use the rank order of the data and in some cases the difference in value rather than the absolute value. This is because it was not always valid to make assumptions about the distribution of the data [16]. Parametric methods require normally distributed data. The Wilcoxon's test is used in situations when individual values for two samples are related. For instance, when the same set of twelve subjects are exposed to two magnitudes of vibration, the two sets of data would be related samples. Usually a confidence level $p$ value of less than 0.05 is used to indicate that the two samples of data are different.

\section{$3 \quad$ Results}

The results are presented in the working order of the MISO recursive operation of one subject (S9), as an example, in Figures 4 to 8. The results for all twelve subjects are presented in Figures 9 to 11, and in Tables 1 and 2.

At $0.125 \mathrm{~ms}^{-2}$ r.m.s. acceleration, an example of the first 6 seconds of the three mathematical inputs and one mathematical output are presented in Figure 4. Note that $x_{3}$ is the sign-conserved square rooted horizontal inline force. Figure 5 shows the power spectral density functions of the three mathematical inputs $G_{11}$ for inline horizontal force, $G_{22}$ for cross-axis vertical force, $G_{33}$ for square rooted inline horizontal force, one mathematical output $G_{y y}$ for inline horizontal excitation acceleration, and the measured lateral y-axis force $\left(G_{\text {lateral }}\right)$ that has been replaced by the third mathematical input $\left(G_{33}\right)$ in the current numerical study. Between 6 and $18 \mathrm{~Hz}, G_{33}$ exhibits a similar profile to the cross-axis vertical force $\left(G_{22}\right)$. 

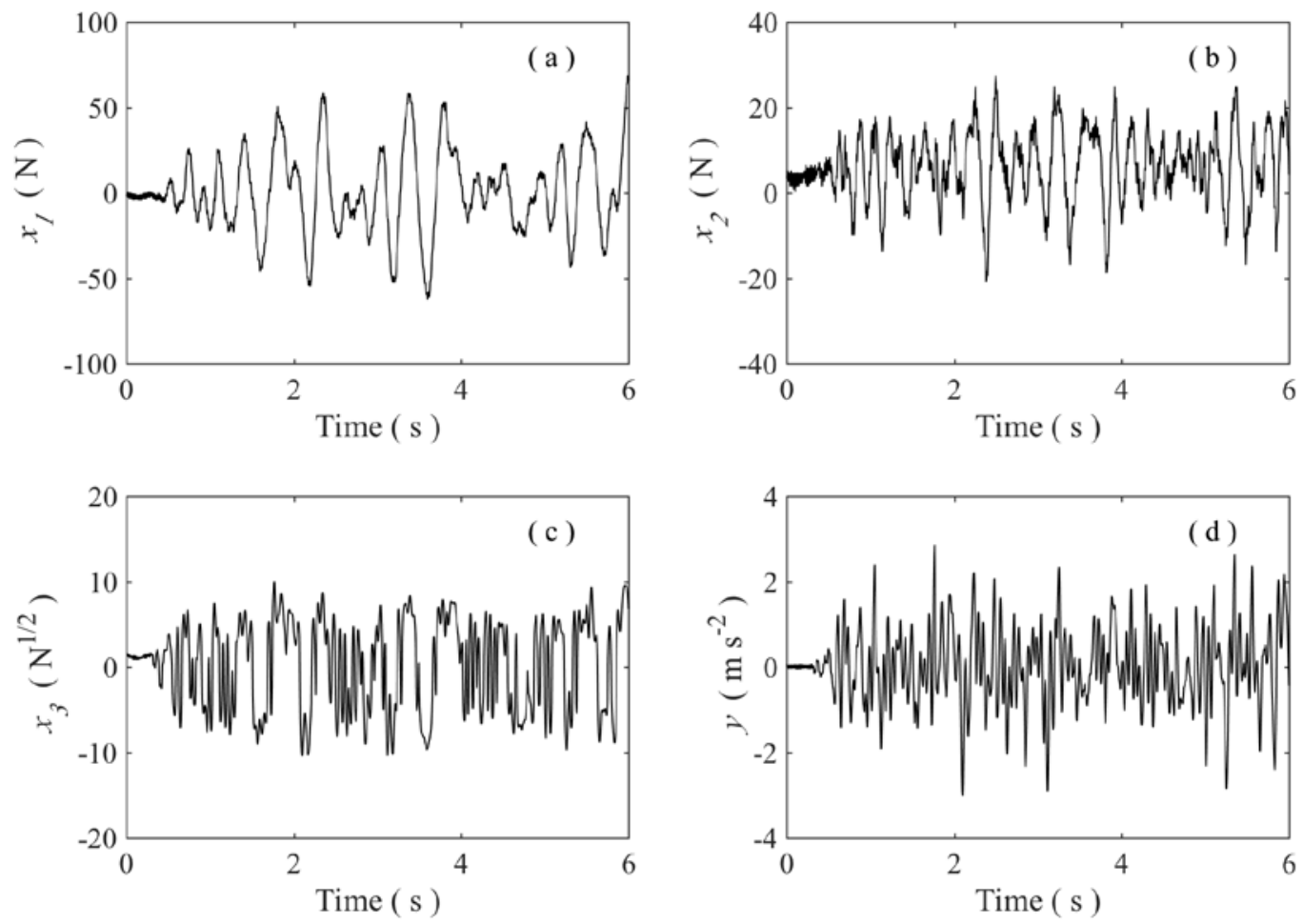

Figure 4 Time histories of the mathematical inputs: (a) $x_{1}$ - horizontal inline output force at the driving point (N); (b) $x_{2}$ - vertical cross-axis output force at the driving point (N); (c) $x_{3}-$ sign conserved square rooted horizontal inline force $\left(\mathrm{N}^{1 / 2}\right)$; and mathematical output: (d) $y$ - horizontal acceleration excitation at the base $\left(\mathrm{ms}^{-2}\right)$ with each lasting for 90 seconds at $1.0 \mathrm{~ms}^{-2}$ r.m.s. for subject S9.

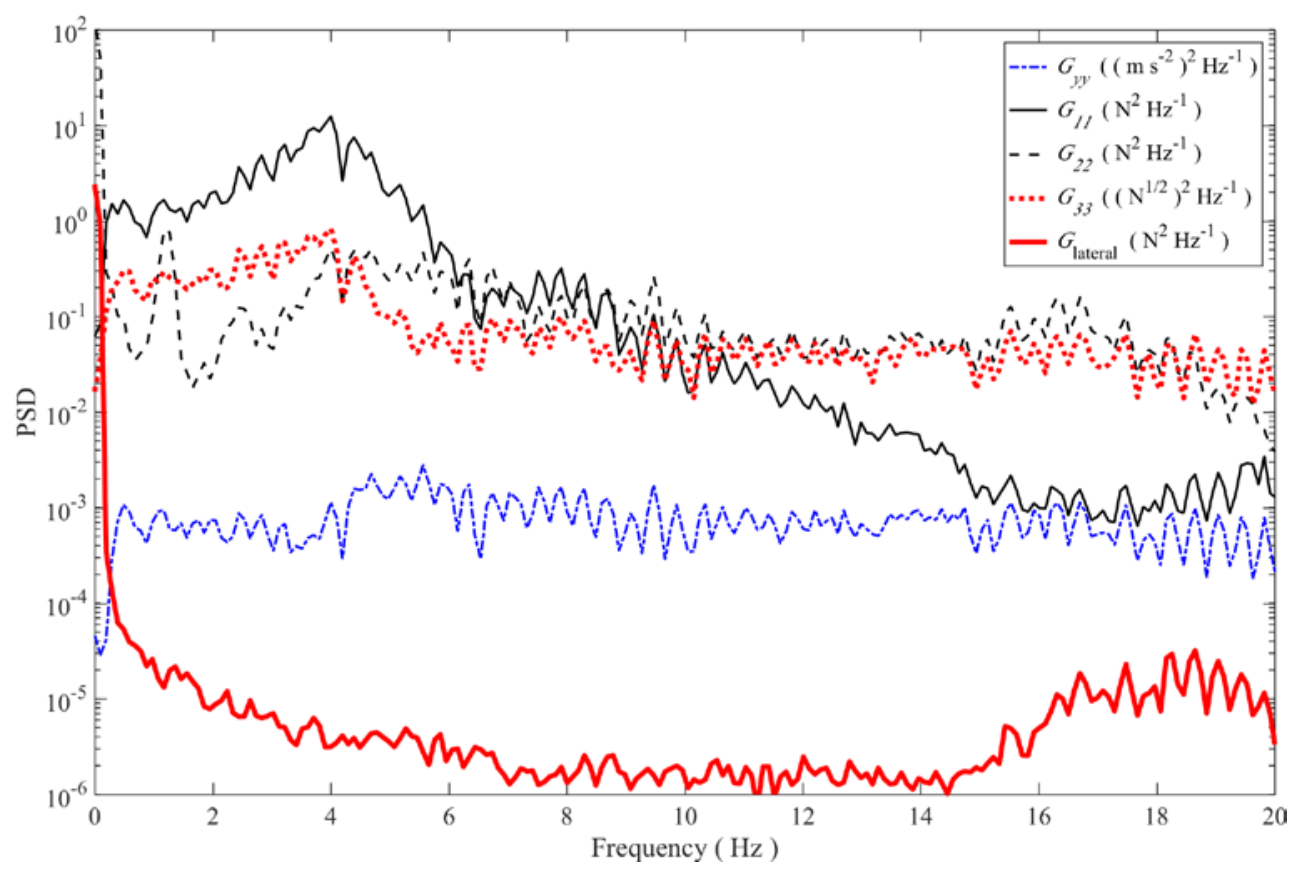

Figure 5 Power Spectral Densities of the excitation acceleration $G_{y y}$, the inline z-axis force $G_{11}$, the cross-axis vertical $x$-axis force $G_{22}$, the sign conserved square root of the inline z-axis force $G_{33}$ and the cross-axis lateral $y$-axis force $G_{\text {lateral }}$ of subject $S 9$ at $0.125 \mathrm{~ms}^{-2}$ r.m.s. 


\subsection{Partial and multiple coherence functions}

The partial and multiple coherence functions, and the corresponding correlated and uncorrelated transfer functions at $0.125 \mathrm{~ms}^{-2}$ r.m.s. acceleration are presented in Figure 6, depicting the working sequence of the recursive conditioning algorithm. The correlated $(H)$ and uncorrelated $(L)$ transfer functions produce envelopes for the inverse of the apparent mass transfer function $\left(H_{1}\right.$ and $H_{2}$ in Figure $7 \mathrm{a})$ due to the reverse path algorithm. The main drop in the ordinary coherence $\left(\mathrm{coh}_{1 y}\right)$ is between 12 and $20 \mathrm{~Hz}$. Shown by its partial coherence ( $\left.\operatorname{coh}_{2 y}\right)$, the second mathematical input $\left(x_{2}\right)$, i.e. the vertical cross-axis output force, exhibits improvement of up to $40 \%$ to the multiple coherence $\left(\mathrm{coh}_{y y}\right)$ in the frequency range where the ordinary coherence drops below $70 \%$. At $0.125 \mathrm{~ms}^{-2}$ r.m.s., the vertical cross-axis force also improves the ordinary coherence at lower frequencies near the primary resonance i.e. around 3, 4 and $7 \mathrm{~Hz}$. The third mathematical input $\left(x_{3}\right)$, i.e. the sign conserved square rooted inline output force, had little contribution to the overall response.

At $1.0 \mathrm{~ms}^{-2}$ r.m.s., the vertical cross-axis force $\left(\operatorname{coh}_{2 y}\right)$ contributes to the multiple coherence ( $\left.\operatorname{coh}_{y y}\right)$ by up to $70 \%$ between 10 and $16 \mathrm{~Hz}$. The third mathematical input of square rooted inline horizontal force $\left(\operatorname{coh}_{3 y}\right)$ also contributes $10 \%$ to $30 \%$ to the multiple coherence in the same frequency range (Figure 8f). This is considerably higher than that obtained at the lower vibration magnitude of 0.125 $\mathrm{ms}^{-2}$ r.m.s. (Figure 8c). The drop in ordinary coherence was observed over a wider frequency range and greater in reduction at the higher vibration magnitude than at the lower magnitude. In the frequency range of the main coherence drop, the cross-axis force improved the multiple coherence more at the higher magnitude of vibration than at the lower magnitude. There was a small but evident improvement in the multiple coherence at lower frequencies, near the primary resonance around 2 to 4 $\mathrm{Hz}$. 

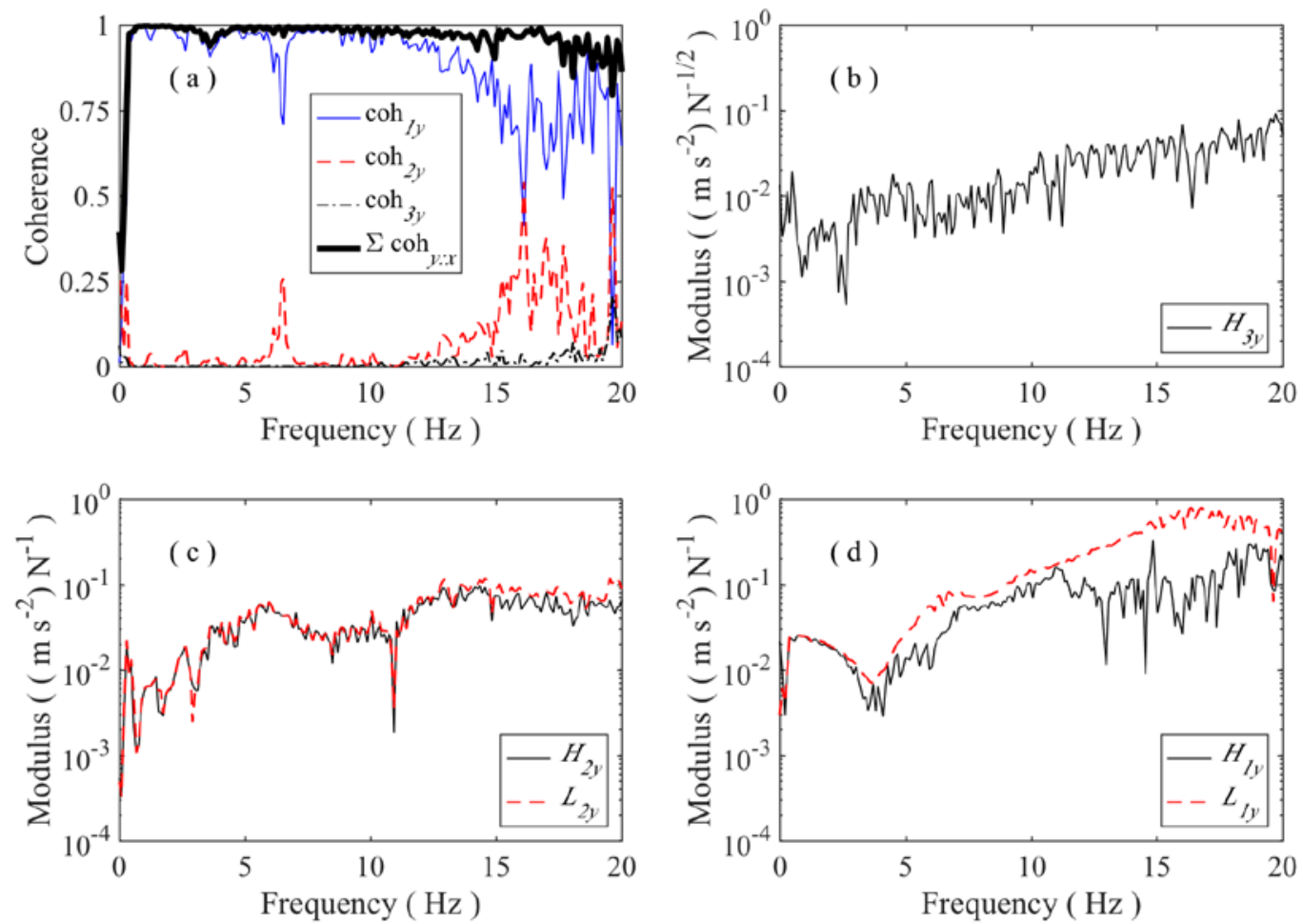

Figure 6 (a) Ordinary coherence $\operatorname{coh}_{1 y}$, partial coherence $\operatorname{coh}_{2 y}$, $\operatorname{coh}_{3 y}$, and multiple coherence $\sum \operatorname{coh}_{y: x}$ computed using individual and combined mathematical inputs $\left(x_{1}, x_{2}, x_{3}\right)$ and output (y). (b) $H_{3 y}$, (c) $H_{2 y}$ and (d) $H_{1 y}$ are FRF based on correlated mathematical inputs. (c) $L_{2 y}$ and (d) $L_{1 y}$ are FRF based on uncorrelated mathematical inputs. Vibration magnitude: $0.125 \mathrm{~ms}^{-2}$ r.m.s., subject S9.

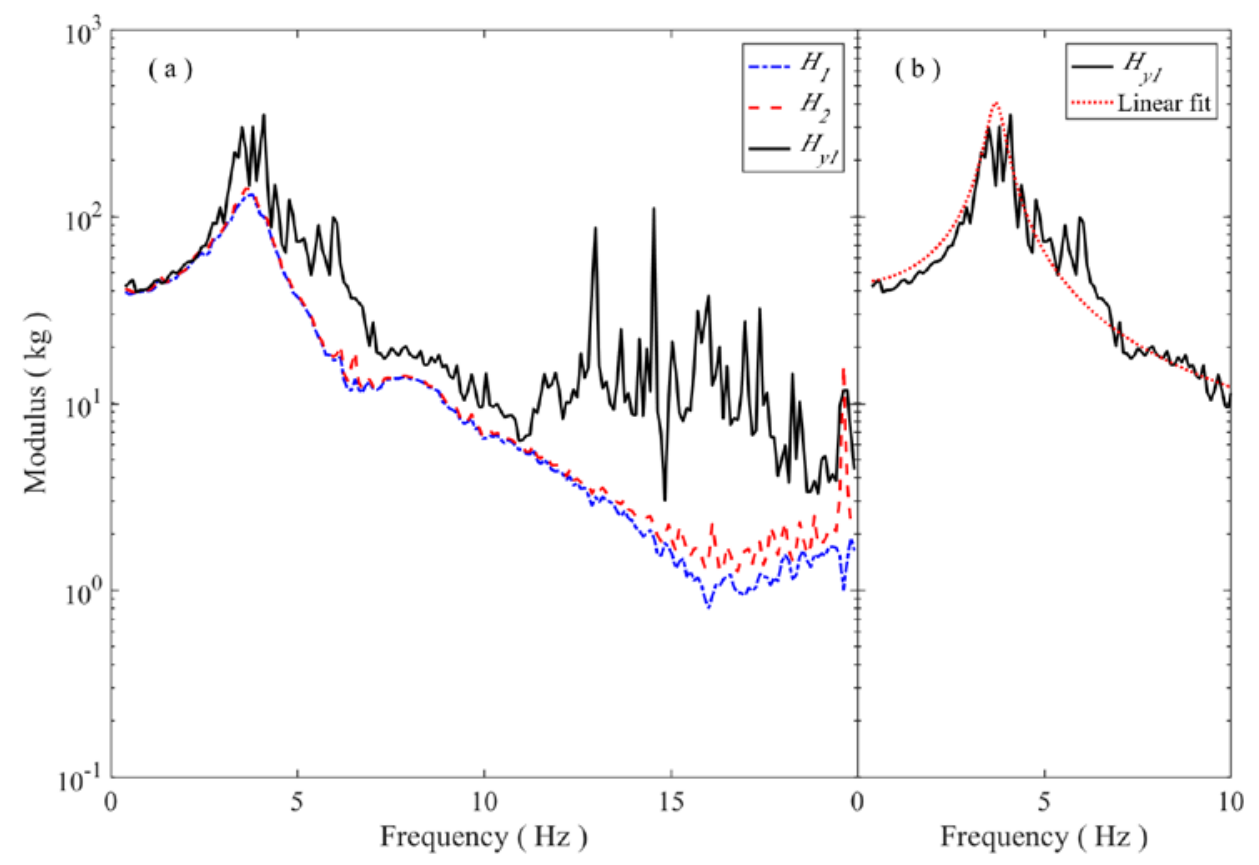




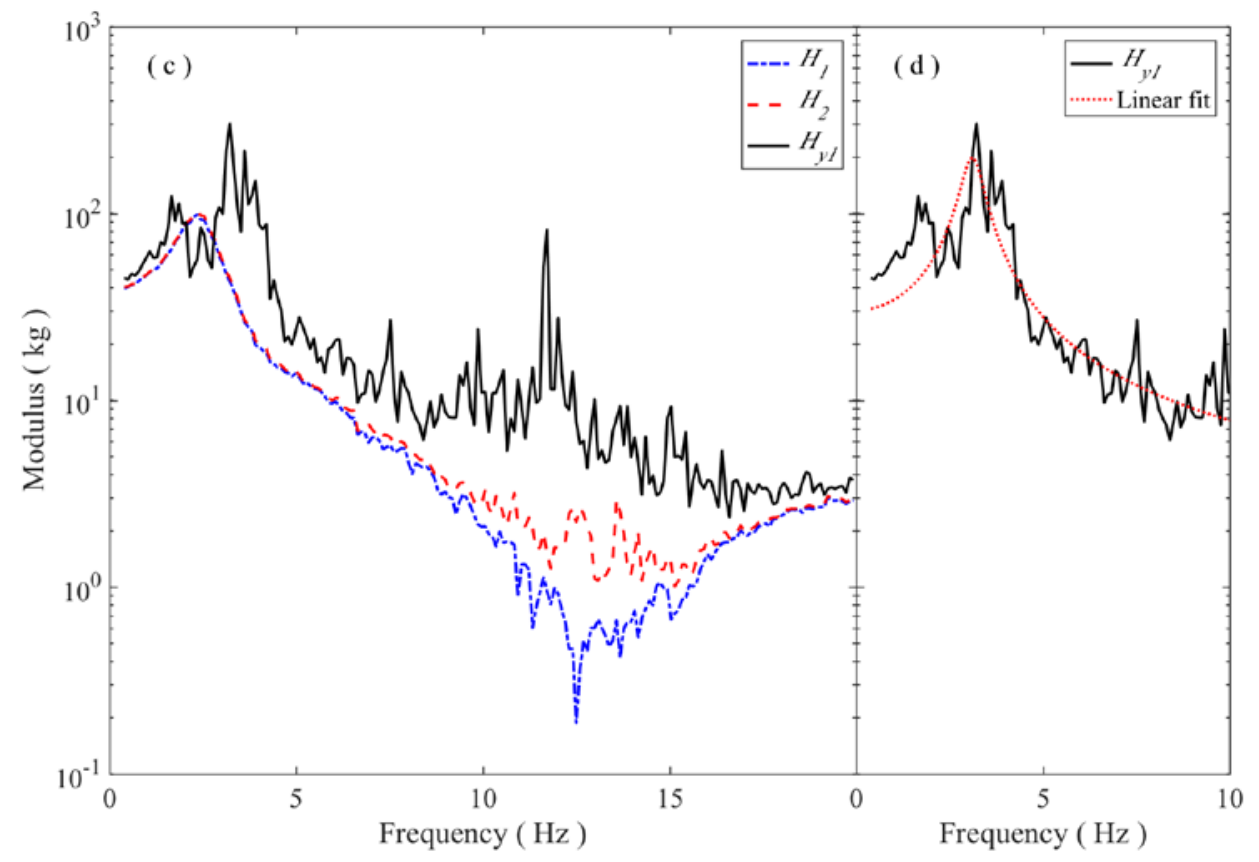

Figure 7 Individual (Subject S9) $H_{1}$ and $H_{2}$ as standard apparent mass FRF, $H_{y 1}$ the linear FRF determined from the mathematical output $y$ and mathematical input $x_{1}$ at vibration magnitude 0.125 $\mathrm{ms}^{-2}$ r.m.s. (a) and (b), and at $1.0 \mathrm{~ms}^{-2}$ r.m.s. (c) and (d). (b) and (d) show the linear FRF 2-poles and 2zeros fit of the $H_{y 1}$ function at the two magnitudes respectively.

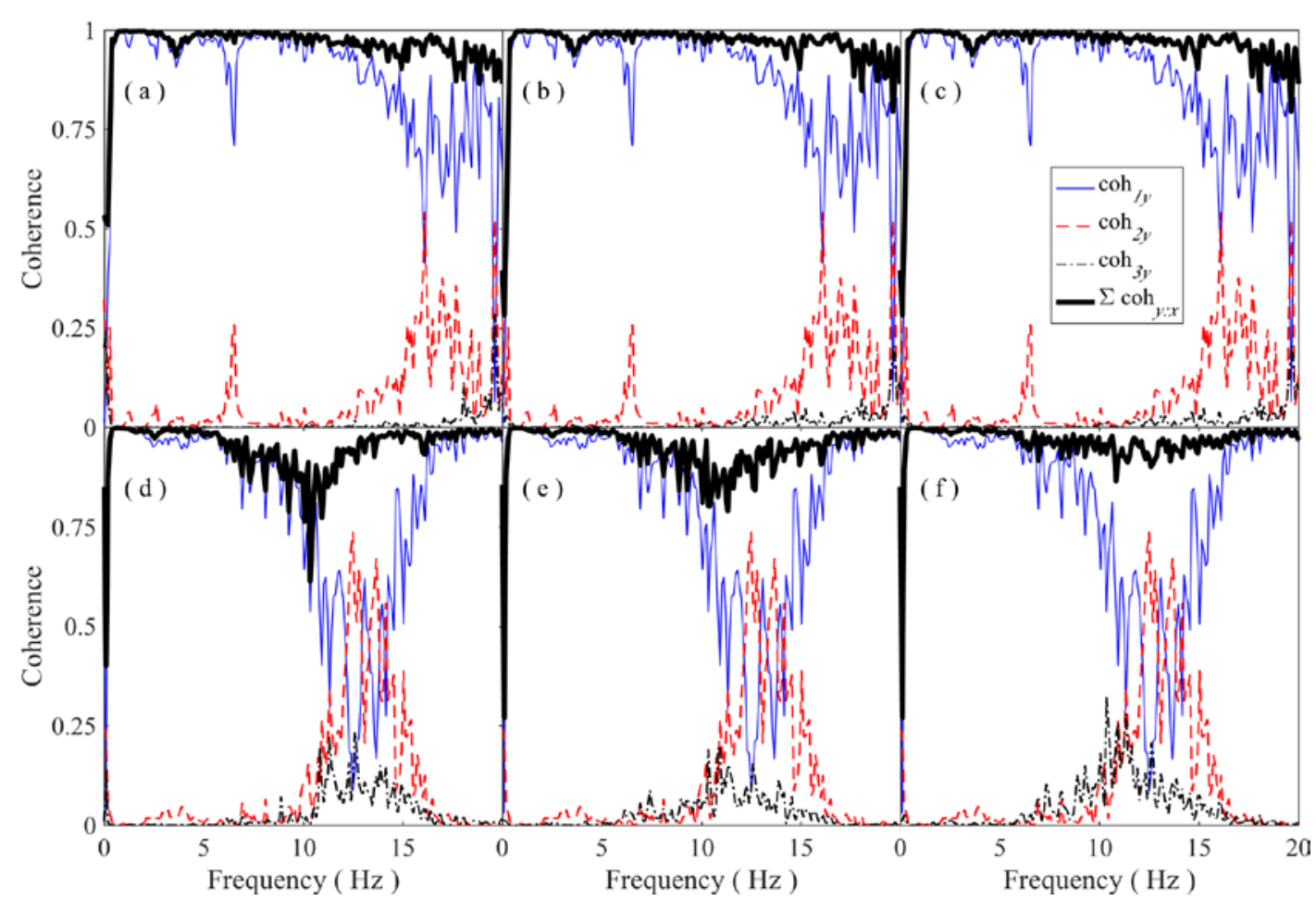

Figure 8 Individual (subject S9) ordinary coherence $\operatorname{coh}_{1 y}$, partial coherence $\operatorname{coh}_{2 y}$, $\operatorname{coh}_{3 y}$, and summed multiple coherence function $\sum \mathrm{coh}_{y: x}$ computed using individual and combined mathematical inputs $\left(x_{1}, x_{2}, x_{3}\right)$ and output $(y)$. (a), (b) and (c) show vibration magnitude at $0.125 \mathrm{~ms}^{-2}$ r.m.s. (d), (e) and (f) show vibration magnitude at $1.0 \mathrm{~ms}^{-2}$ r.m.s. (a) and (d) use the original lateral force for 
mathematical input $x_{3}$. (b) and (e) use the squared inline force $x_{1}$ as $x_{3}$. (c) and (f) uses the sign conserved square root of the inline force $x_{1}$ as $x_{3}$.

\subsection{Ordinary and conditioned frequency response functions}

The difference between the two standard FRF $H_{1}$ and $H_{2}$ of an individual subject (S9) in Figure 7 illustrates the frequency range at which the ordinary coherence function is low.

The peak frequencies of the ordinary $\left(H_{1}\right)$ and conditioned $\left(H_{y 1}\right)$ FRF at the two vibration magnitudes are summarised in Table 1 and presented in Figure 9. The ordinary FRF peak frequencies were obtained by fitting a parallel two-degree-of-freedom mass-spring-damper lumped parameter model to $H_{1}$ by Huang and Griffin [1]. The conditioned FRF peak frequencies are estimated by fitting a twozero-two-pole continuous filter in the Laplace form to $H_{y 1}$ using the MATLAB routine 'invfreqs' in the present study. The $H_{y 1}$ obtained from the reverse path algorithm exhibits much more scattered variation in the frequency range 0.5 to $20 \mathrm{~Hz}$. Therefore, the two-zero-two-pole filter does not provide as satisfactory a fit as the lumped parameter fit for the ordinary $H_{1}$. At $1.0 \mathrm{~ms}^{-2}$ r.m.s., there is a significant difference between the peak frequencies estimated from the $H_{y 1}$ fit and those estimated from the $H_{1}$ fit $(p<0.05$, Wilcoxon matched-pairs signed ranks test, Table 2$)$. In contrast, no statistically significant difference in the peak frequencies was found between the two techniques at $0.125 \mathrm{~ms}^{-2}$ r.m.s. ( $p>0.05$, Wilcoxon). The magnitude dependent biomechanical nonlinearity is observed using both the $H_{1}$ fit method and the $H_{y 1}$ fit method - the peak frequencies are significantly different at the two vibration magnitudes ( $p<0.05$, Wilcoxon).

Table 1 Peak frequencies $\left(f_{r}\right)$ at low $\left(0.125 \mathrm{~ms}^{-2}\right.$ r.m.s. $)$ and high $\left(1.0 \mathrm{~ms}^{-2}\right.$ r.m.s. $)$ acceleration magnitudes. Peak frequencies for ordinary FRF $H_{1}$ are obtained by fitting a two-degree-of-freedom lumped parameter mass-spring-damper system in the frequency range 0 to $10 \mathrm{~Hz}$ by Huang and Griffin [1]; peak frequencies for the conditioned FRF $H_{y 1}$ in the present study are obtained by fitting a two-zero-two-pole Laplace FRF estimation in the frequency range 0 to $10 \mathrm{~Hz}$. Both fitting processes use least square error optimisation.

\begin{tabular}{c|cccccccccccc}
\hline \multicolumn{1}{c|}{$f_{r}(\mathrm{~Hz})$} & $\mathrm{S} 1$ & $\mathrm{~S} 2$ & $\mathrm{~S} 3$ & $\mathrm{~S} 4$ & $\mathrm{~S} 5$ & $\mathrm{~S} 6$ & $\mathrm{~S} 7$ & $\mathrm{~S} 8$ & $\mathrm{~S} 9$ & $\mathrm{~S} 10$ & $\mathrm{~S} 11$ & $\mathrm{~S} 12$ \\
\hline Low $H_{1}$ & 3.81 & 3.32 & 4.00 & 3.32 & 3.81 & 3.81 & 3.42 & 4.00 & 3.71 & 3.61 & 3.52 & 3.52 \\
\hline Low $H_{y 1}$ & 3.81 & 2.54 & 2.54 & 3.32 & 4.39 & 3.81 & 3.13 & 4.39 & 3.71 & 3.22 & 3.42 & 3.42 \\
\hline High $H_{1}$ & 2.44 & 2.15 & 2.73 & 2.25 & 2.54 & 2.54 & 2.44 & 2.64 & 2.34 & 2.34 & 2.15 & 2.44 \\
\hline High $H_{y 1}$ & 2.44 & 2.64 & 2.93 & 3.22 & 3.32 & 2.54 & 3.52 & 3.32 & 3.13 & 2.15 & 3.22 & 2.44 \\
\hline
\end{tabular}


Table 2 Statistical significance of the ordinary and conditioned apparent mass FRF resonance frequencies $\left(f_{r}\right.$ in $\left.\mathrm{Hz}\right)$ at the low and high magnitudes ( $p$ values for Wilcoxon matched-pairs signed ranks test, * indicates $p<0.05$ and the results are significantly different)

\begin{tabular}{c|cccc}
\hline & Low $H_{1}$ & Low $H_{y 1}$ & High $H_{1}$ & High $H_{y 1}$ \\
\hline Low $H_{1}$ & - & 0.292 & $0.002^{*}$ & $0.003^{*}$ \\
Low $H_{y 1}$ & - & - & $0.003^{*}$ & $0.025^{*}$ \\
High $H_{1}$ & - & - & - & $0.011^{*}$ \\
High $H_{y 1}$ & - & - & - & - \\
\hline
\end{tabular}
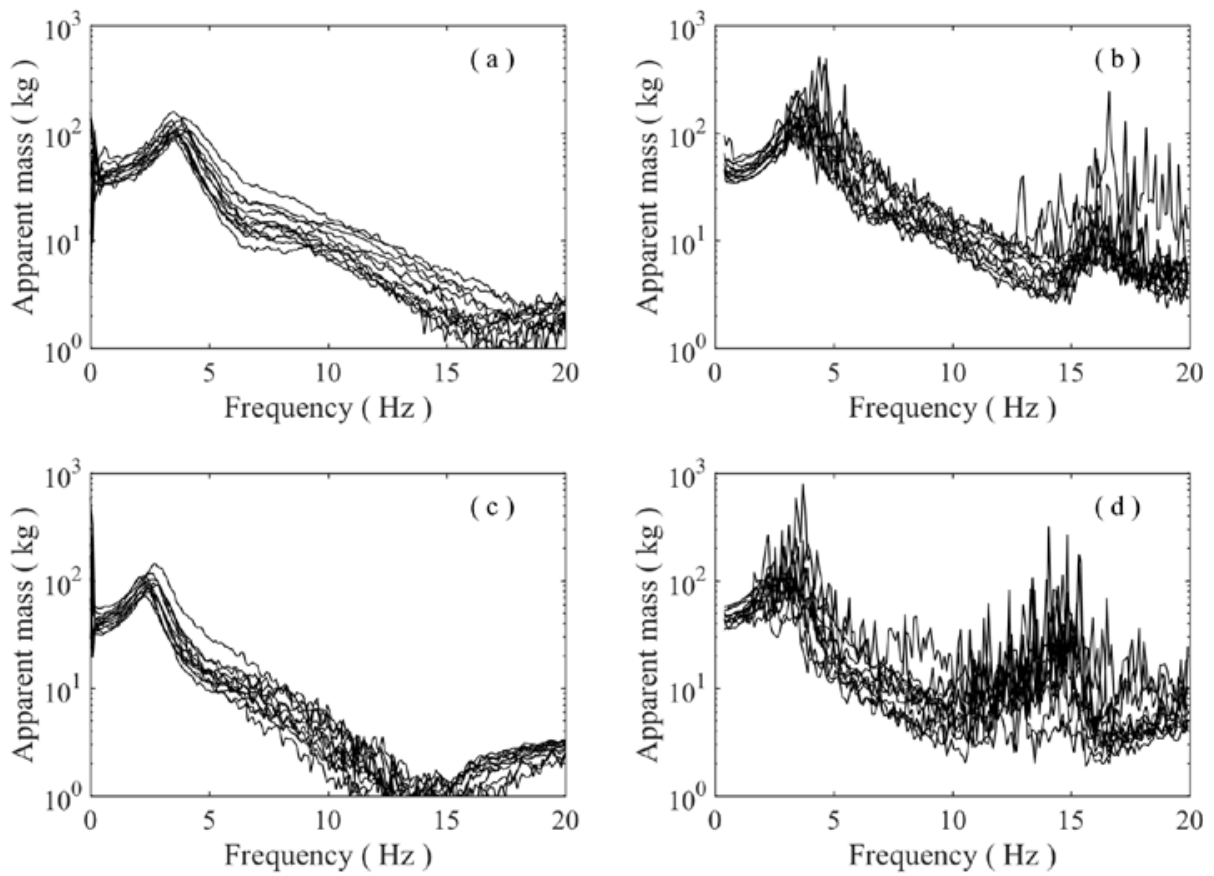

Figure 9 Individual apparent mass estimated by ordinary $H_{1}$ FRF for 12 subjects at $0.125 \mathrm{~ms}^{-2}$ r.m.s. (a) and $1.0 \mathrm{~ms}^{-2}$ r.m.s. (c), and then estimated by conditioned $H_{y 1}$ FRFs for 12 subjects at 0.125 $\mathrm{ms}^{-2}$ r.m.s. (b) and $1.0 \mathrm{~ms}^{-2}$ r.m.s. (d).

The peak frequencies only depict dynamic behaviour around the resonance peak. The individual $H_{1}$ and $H_{y 1}$ at both magnitudes for all twelve subjects provide a sense of inter-subject variation over the entire frequency range from 0.5 to $20 \mathrm{~Hz}$ (Figure 9). For the ordinary $H_{1} \mathrm{FRF}$, there was greater variation around the resonance frequencies between 0.5 and $5 \mathrm{~Hz}$ at the lower magnitude of $0.125 \mathrm{~ms}^{-2}$ r.m.s. than those at $1.0 \mathrm{~ms}^{-2}$ r.m.s. (Figure 9 a and c). However, between 10 and $20 \mathrm{~Hz}$ where the major 
coherence drop occurred, the variation was greater at the higher magnitude. For the conditioned $H_{y 1}$ FRF, there was greater variation in the apparent mass at the higher magnitude of $1.0 \mathrm{~ms}^{-2} \mathrm{r}$.m.s. across the frequency range 0.5 to $20 \mathrm{~Hz}$ (Figure $9 \mathrm{~b}$ and d). Between 0.5 and $10 \mathrm{~Hz}$ where the primary resonance occurs, the $H_{y 1}$ estimate shows greater variation than the $H_{1}$ estimate. Some subjects exhibit greater variation than others. The summed multiple coherence is improved.

\subsection{Statistical characteristics of the response forces}

The skewness, a measure of asymmetry, and kurtosis, a measure of whether the data has increased or reduced 'tails' relative to a Gaussian normal distribution, for each time history (90 seconds) for twelve subjects at each of 0.125 and $1.0 \mathrm{~ms}^{-2}$ r.m.s. are compared in Figure 10. The kurtosis value of 3 for a Gaussian distribution was always subtracted from any kurtosis values presented. The horizontal z-axis excitation acceleration shows greater asymmetry and heavier tails compared to a normally distributed random set at the higher vibration magnitude. This would be the benchmark to be compared with the three output forces in $x, y$ and $z$ axes. For a perfectly 'linear' system, one would expect that the same amount of skewness and kurtosis would appear on each of the output forces. To ease the comparison between the three response forces and the horizontal excitation acceleration, the skewness and kurtosis values of the excitation acceleration (Figure $10 \mathrm{a}$ and b) were subtracted from the skewness and kurtosis values presented for the three response forces in $x, y$ and $z$ axes (Figure $10 \mathrm{c}$ to $\mathrm{h}$ ). These are therefore referred to as centred skewness and centred kurtosis.

The asymmetry and tail characteristics of the distribution could be used to interpret the response forces relative to the characteristics of the excitation acceleration. A large skewness indicates that some other factor or input might have contributed a bias or greater range of force response given that the underlying nonlinearity sought after is similar in all subjects. A negative skewness value would imply that there exists an increased tail of values below the mean or that the values above the mean are concentrated in a smaller range or interval. If the kurtosis values are lower than zero, there is an increased chance that the sprung mass of the body is regularly reaching the 'end-stops' of the anatomical boundaries. A high kurtosis value above zero would indicate either 1) effects that might have caused the response force to deviate from a normal tail, or 2) a low level of response force where the chance of biased non-normal behaviour would emerge e.g. in the $x$-axis and $y$-axis forces.

One of the twelve subjects, S3, showed unusually high skewness and kurtosis values for the horizontal $z$-axis inline force $\left(x_{1}\right)$ and vertical $x$-axis cross-axis force $\left(x_{2}\right)$ at $0.125 \mathrm{~ms}^{-2}$ r.m.s. An inspection of the time histories revealed that this was likely due to drifts over time of the charge amplifier of the forcesensing instrument. The discussion will therefore focus on the remaining eleven subjects.

For the horizontal z-axis inline force, its symmetry is similar to that of the low magnitude excitation acceleration for 11 out of 12 subjects except for S3 (Figure 10 a and c). At the high vibration magnitude, 9 out of the 12 subjects showed an asymmetry in the opposite direction to that of the 
excitation acceleration. At the lower magnitude, 11 subjects show reduced tails than the excitation acceleration except for subject S3 (Figure $10 \mathrm{~b}$ and d). At the higher magnitude, 5 out of 12 subjects showed reduced tails. A reduced tail might indicate that the sprung mass of an oscillation system has regularly reached the 'end-stops' boundaries of its motion giving rise to forces that do not follow a Gaussian distribution.

For the $x$-axis cross-axis force, there is slightly higher level of asymmetry than the $z$-axis force at the low magnitude, but slightly lower level of asymmetry than the z-axis force at the high magnitude (Figure $10 \mathrm{c}$ and e). There is a slightly reduced tail for the low magnitude but a considerable reduction in tail size for the high magnitude acceleration (Figure $10 \mathrm{~d}$ and $\mathrm{f}$ ).

For the $y$-axis cross-axis force, the level of asymmetry is very similar to the excitation at both magnitudes (Figure $10 \mathrm{~g}$ ). The tails are much reduced, corresponding to a negative change in the kurtosis, for both magnitudes (Figure $10 \mathrm{~h}$ ).
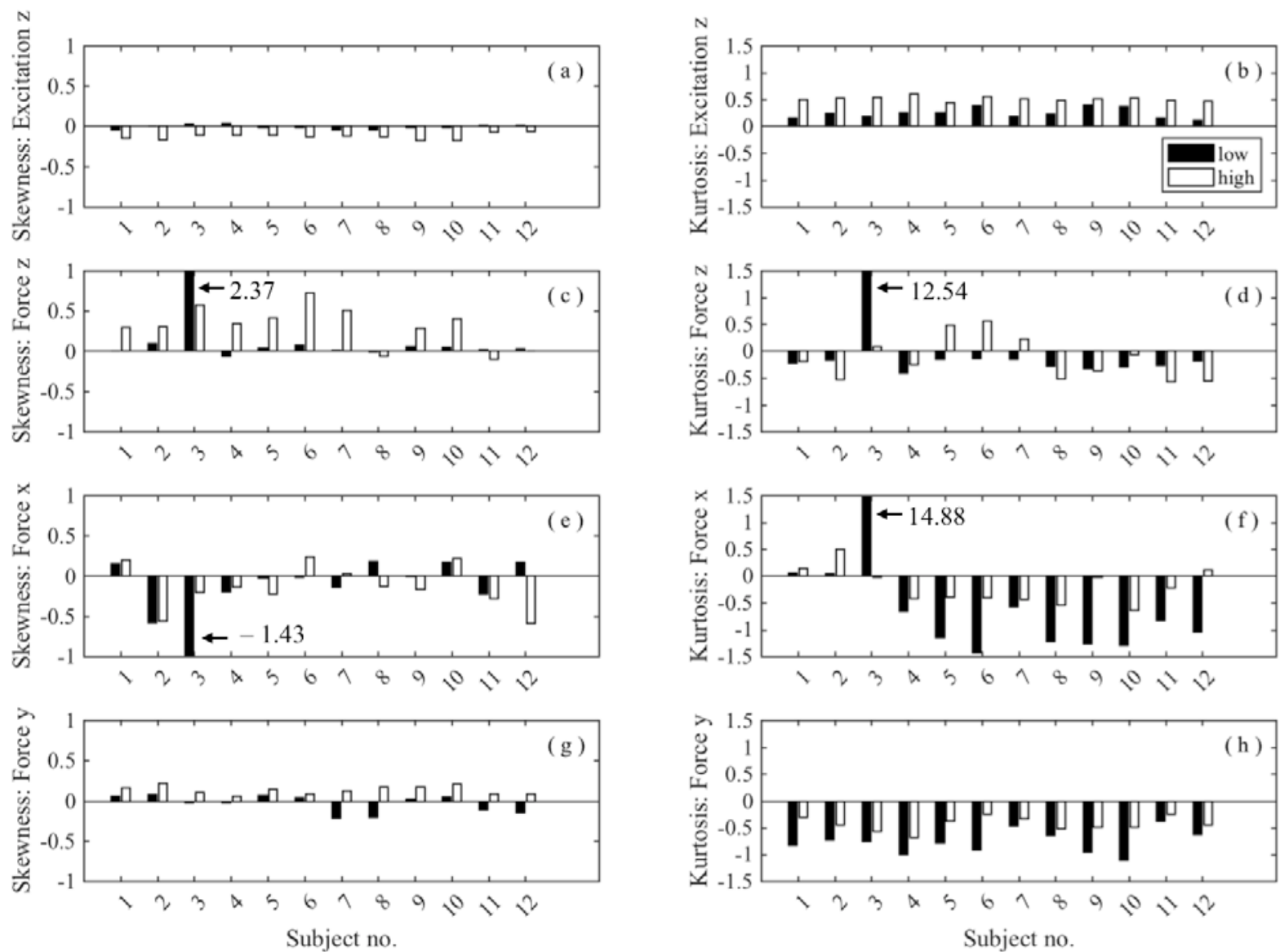

Figure $10 \quad$ Skewness (a) and kurtosis (b) values of the $z$-axis excitation acceleration, centred skewness and kurtosis of inline $z$-axis force (c and d), centred skewness and kurtosis of fore-and-aft cross $x$-axis force (e and f), and centred skewness and kurtosis of lateral cross $y$-axis force ( $\mathrm{g}$ and $\mathrm{h}$ ) time histories of 90 seconds at the low magnitude of $0.125 \mathrm{~ms}^{-2}$ r.m.s. and the high magnitude of 1.0 $\mathrm{ms}^{-2}$ r.m.s. of 12 subjects. Subject 3 is identified in c, d, e, and f. 


\subsection{Partial and multiple coherence functions}

The partial coherence of the vertical cross-axis force obtained by the recursive conditioning procedure improved the overall multiple coherence representing the linearity of the dynamic body system in the frequency range 8 to $20 \mathrm{~Hz}$. However, in the frequency 8 to $18 \mathrm{~Hz}$, some compensated coherences were about 0.9 at $0.125 \mathrm{~ms}^{-2}$ r.m.s and around 0.7 at $1.0 \mathrm{~ms}^{-2}$ r.m.s. There must have been some other causes. Huang and Griffin [1] speculated that the low output force at the driving point, or, high noise and distortion, at these frequencies could be the primary cause. By constructing the mathematical input of the 'reversed' dynamic system, the current study intends to provide a more quantitative explanation of the coherence drop in the horizontal apparent mass. By replacing the $y$-axis lateral cross-axis force by the sign conserved square rooted $z$-axis horizontal inline force, as the third mathematical input in the reversed recursive conditioning procedure, the coherence was further improved at the higher magnitude of vibration, but was not so apparent at the lower magnitude (Figure 8 a and d). The increased contribution from the third mathematical input at the higher magnitude seems to agree with a previous suggestion that the body system is more nonlinear at the higher excitation magnitude [2]. Alternative nonlinear quantities could also be considered in the analysis, if required, or considered to be based on biomechanical principles.

The constructed mathematical input $\left(x_{3}\right)$ played a relatively small role in the multiple coherence function in comparison to the main cross-axis response force in the vertical $x$-axis of the recumbent subject. The cross-axis movement of the recumbent human body introduced complexity when analysing the linearity or the nonlinearity of the body. Any test conditions involving human subjects will involve degrees of cross-axis response. Means to eliminate or control such movements in experiments are desirable.

By mathematically constructing analytical inputs, comparison between the effects of physical inputs, i.e. $x_{1}$ and $x_{2}$, and analytical inputs, i.e. $x_{3}$, of the reversed system is now possible (Figure 8). The constructed input $\left(x_{3}\right)$ herein this paper could be either the square of the first mathematical (physical) input $x_{1}$ (Figure $8 \mathrm{~b}$ and e), the sign conserved square root of $x_{1}$ (Figure $8 \mathrm{c}$ and $\mathrm{f}$ ), or the original physical lateral $y$-axis cross-axis force (Figure 8 a and d). At the low vibration magnitude, there was not much difference by changing between the three constructed inputs. But at the higher magnitude, the sign conserved square root component seemed to offer the highest contribution in the multiple coherence for the third mathematical input $x_{3}$ (Figure $8 \mathrm{~d}$, e and f). Similar behaviour was observed across the twelve subjects. This was the primary reason for the current study to use the sign conserved square root construction. 
The higher coherence using a square root function of the force, rather than that using a squared function, suggest that the nonlinear component is better captured by an increased force response for a given input displacement or acceleration, rather than a reduced force response for the same input displacement or acceleration. As the force increases, the sign conserved square root function produces an increased value, but with a decreasing gradient as a function of the input acceleration. The reducing force behaviour coincides with the biomechanical nonlinearity observations at the main resonance frequencies; with higher magnitudes of vibration the resonance frequency for the apparent mass decreases indicating a reducing force dynamic response.

Before conducting the reversed MISO process, the order of the mathematical inputs could be determined using the ordinary FRF [9]. The inline response usually dominates when the effect of active muscular activity is considered secondary or less important. The majority of whole-body vibration studies suggest that the primary cross-axis response during a single axis excitation would be in the mid-sagittal plane. Among the three orthogonal axes fixed to the recumbent human body (Figure 1 ), the horizontal $z$-axis inline force was set as the first mathematical input $\left(x_{1}\right)$, and the vertical $x$-axis cross-axis force was the second mathematical input $\left(x_{2}\right)$. By treating the third mathematical input $\left(x_{3}\right)$ as the lateral y-axis cross-axis force and then as an analytically constructed input, the comparison can reveal the effective or better mathematical representation of the nonlinear component in a system.

\subsection{Vibration magnitude and peak frequency}

By relating the peak frequencies estimated at the two vibration magnitudes, 0.125 and $1.0 \mathrm{~ms}^{-2}$ r.m.s., and overlaying results from the two analytical methods, $H_{1}$ fit and $H_{y 1}$ fit, the gradients of trend lines for each method would reveal the degree of a numerical 'linearity' (Figure 11). A gradient of one for this plot, indicated by a $45^{\circ}$ line on Figure 11, would indicate a perfectly linear relationship. As the peak frequency at the low vibration magnitude increases or decreases its counterpart at the high vibration magnitude would also change by the same amount. 


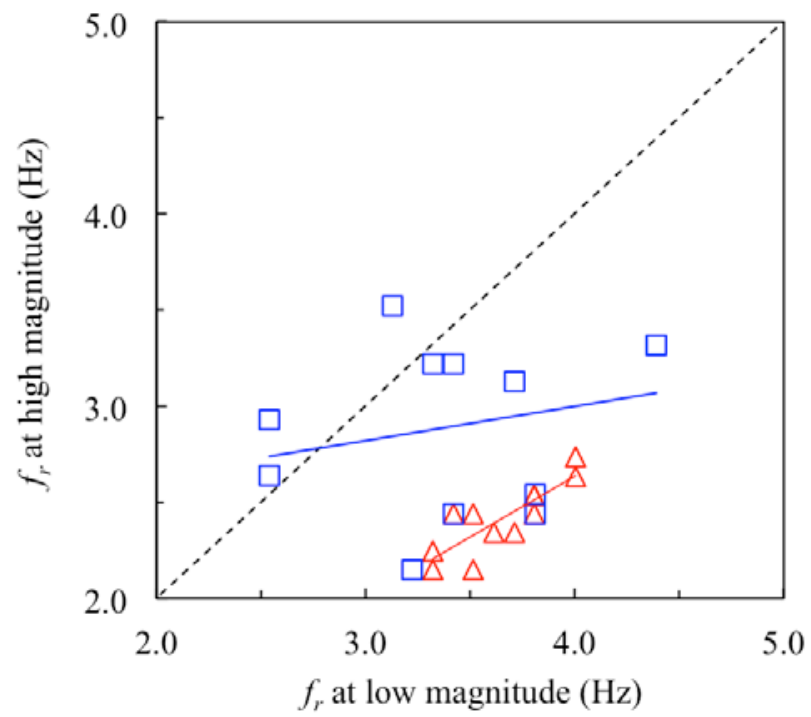

$$
\begin{array}{|l}
\hline \quad H_{l} \\
\square \quad H_{y l} \\
-\quad y=x \\
\square \quad \text { Linear fit } H_{l} \\
\square \quad \text { Linear fit } H_{y l} \\
\mathrm{y}=0.18 \mathrm{x}+2.29 \\
\mathrm{R}^{2}=0.06 \\
\mathrm{y}=0.63 \mathrm{x}+0.11 \\
\mathrm{R}^{2}=0.70
\end{array}
$$

Figure 11 Comparison of the peak frequencies $\left(f_{r}\right)$ at low $\left(0.125 \mathrm{~ms}^{-2}\right.$ r.m.s. $)$ and high $\left(1.0 \mathrm{~ms}^{-2}\right.$ r.m.s.) magnitudes using: 1 ) ordinary FRF $H_{1}$ (solid triangle) obtained by fitting to a two-degree-offreedom lumped parameter mass-spring-damper system in the frequency range 0 to $10 \mathrm{~Hz}$ [1] with linear trend line slope $=0.63$ and $\mathrm{R}^{2}=0.70 ; 2$ ) conditioned FRF $H_{y 1}$ (square) obtained by fitting to a 2zero-2-pole Laplace FRF estimation in the frequency range 0 to $10 \mathrm{~Hz}$ with linear trend line gradient = 0.18 and $\mathrm{R}^{2}=0.06$. The diagonal dotted line represents theoretical equal peak frequencies for the individual subjects at the two input acceleration magnitudes.

With a coefficient of determination $\mathrm{R}^{2}$ of 0.70 , a linear trend line produced a reasonable goodness of fit for the ordinary $H_{1}$ fit of the peak frequencies of the twelve subjects. With a gradient of 0.63 , the $H_{1}$ fit method indicates that the peak frequency is approximately reduced by a third compared to the peak at the lower acceleration input level across the twelve subjects.

With a $\mathrm{R}^{2}$ of 0.06 , the conditioned $H_{y 1}$ fit of peak frequencies is largely unpredictable by a linear trend line. This was partly due to the noisy conditioned FRF $H_{y 1}$ meaning it was difficult to identify a peak. The two-zero-two-pole linear filter might not be a good characterisation model for the uncorrelated $H_{y 1}$ function (Figure 11). Bearing in mind the poor quality of fit, the highly scattered estimates by $H_{y 1}$ might imply that the underlying dynamic systems of the human body are fundamentally different between each subject. A change in excitation acceleration magnitude will cause very different changes in the resonance behaviour. Some subjects will change more than the others.

The ordinary FRF estimate $H_{1}$ fit approach assumes a linear system between the inputs and output, without consideration of the interaction or correlation between the input channels. Whilst it is acceptable for systems exhibiting linearity and high coherence, it is less justifiable as the coherence reduces and potentially the behaviour is clearly not linear. Analysis of the human biomechanical response would therefore benefit from examination of the underlying uncorrelated linear systems using the reverse path method. The highly variable conditioned FRF $\left(H_{y 1}\right)$ unveil the inter-subject characteristics during exposure to different vibration magnitudes. The approach could be readily 
utilised to investigate other variables such as vibration spectra, vibration waveform, subject posture and subject anthropometric variables.

The multiple excitation magnitudes peak frequency plot, shown in Figure 11, provides a straightforward qualitative comparison of resonance behaviour between 1) different vibration magnitudes and 2) different measures of linearity between the 'smeared' linear estimate $\left(H_{1}\right)$ and the uncorrelated 'linearised' estimate $\left(H_{y 1}\right)$ of the same dynamic system. Although the zero-pole filter is inadequate to fit the $H_{y 1}$ estimate, it demonstrates the large variation of each conditioned input. The human body system is complex with many degrees of freedom. The interpretation of the conditioned estimates will require further investigation using multiple degree of freedom mechanistic models to establish the numerical relationship between the conditioned mathematical inputs and output.

\subsection{Statistical distribution of the response forces}

The primary coupling mechanism between the recumbent person and the excitation surface was the soft tissue at the back of the subject in a 'shearing' mode in the $z$-axis. Due to distinctive anatomical characteristics towards the head and towards the feet, it is plausible that the main 'sprung' body mass coupled on top of the back soft tissue will be subjected to asymmetric elastic and damping restoring forces. The constraining boundaries or 'end-stops' during a cycle of oscillation would also be different. The centred kurtosis values of the response forces would be below zero (Figure $10 \mathrm{c} \mathrm{to} \mathrm{h}$ ), if the sprung mass of the body regularly reaches the 'end-stops' of the anatomical boundaries. This is likely when the vibration magnitude is high.

The intention of the statistical distribution analysis was to provide a qualitative summary of the response force time histories 'centred' relative to the excitation acceleration time histories.

At the low magnitude, the $z$-axis inline force exhibited an overwhelmingly near-zero skewness (except for S3) indicting a symmetric response towards the head and the feet (Figure $10 \mathrm{c}$ ). Asymmetry was evident with 9 out the 12 subjects at the high magnitude. This might be due to asymmetric response towards the head and the feet at higher magnitudes of excitation where anatomical end-stops were reached.

Slightly reduced tails (kurtosis values) can be observed for 11 of the 12 subjects in the $z$-axis inline force at the low magnitude, implying a close-to-normal tail behaviour (Figure $10 \mathrm{~d}$ ). At the high magnitude, 7 of 12 subjects showed reduced tails, and 3 of 12 increased tails. Five out of the 12 subjects (S2, S8, S9, S11, S12) showed more reduced tails at the high magnitude than those at the low magnitude. This does not confirm the original expectation that higher input acceleration levels produce lighter response distribution tails due to more likely access to the end-stop region. These distributions suggest that the response forces at the two excitation magnitudes may have engaged different coupling mechanisms. For example, at the low magnitude, the sprung mass might already have regular access to the anatomical end stops with the response primarily in the inline axis. At the high magnitude, more 
response transfers to measureable cross-axis response, which accommodates the large movement of the sprung mass. The inline response force in this case would have less chance to reach the newly established anatomical end stops that have been modified by increased cross-axis response and possibly a change in property of soft tissue in the excitation-subject interface. Such speculation would be best facilitated by numerical simulation of possible mechanisms. Previous lumped parameter approaches seemed inadequate to represent both the magnitude dependency and the cross-axis coupling mechanism $[3,4,17]$. The present results provide an analytical basis and interpretation.

The $x$-axis cross-axis force showed a similarly low degree of asymmetry at both magnitudes for different subjects (Figure 10 e). The $y$-axis cross-axis force exhibits similar symmetry to the excitation acceleration at both magnitudes (Figure $10 \mathrm{~g}$ ).

A much more reduced tail is present at the lower magnitude than that at the higher magnitude (Figure $10 \mathrm{f}$ ). The different cross-axis coupling mechanisms at high and low magnitudes offer one possible explanation. Another reason could be the low level of response force at the lower magnitude, giving rise to a biased response with reduced tail. There are very similar tail characteristics in both the $x$-axis and $y$-axis for both magnitudes. In the $y$-axis, the low level of the response force seems to be a particular plausible explanation for the much reduced tail.

\section{$5 \quad$ Conclusions}

The analytical study of previously published force data of human response to vibration offers interpretation based on a few classic mathematical tools for dynamics systems that have not been reported before. Very often, the complex human body biomechanical responses are represented by linear frequency response function estimates $\left(H_{1}\right.$ or $\left.H_{2}\right)$ and sometimes further simplified by lumped parameter approach. Such representation tends to smear out the distinction between individuals. The conditioned reverse path FRF (e.g. $H_{y 1}$ ) reveals that uncorrelated 'linear' relationship between physical input (acceleration) and outputs (inline and cross-axis forces) has much greater variation around the primary resonance frequency between 0.5 and $5 \mathrm{~Hz}$. By reversing the input and outputs of the physical system, it is possible to assemble mathematical inputs from the physical output forces and mathematical constructs (e.g. square root of inline force). Depending on the specific construct, this could improve the summed multiple coherence at frequencies where the response magnitude is low. In the present case this is between 6 and $20 \mathrm{~Hz}$. The centred skewness and kurtosis of response forces suggest that there are potential anatomical 'end-stops' for the sprung mass in the inline axis. Different coupling mechanisms might also be present at different magnitudes of vibration. 


\section{Appendix A}

\section{General arithmetic for formulation of the MISO system (Bendat and Piersol, 1993)}

\section{Subscripts:}

1, 2, 3 or $x_{1}, x_{2}, x_{3}$ - mathematical input $y$-mathematical output

i, $o$ - input and output

$q$ - number of inputs

$i, j$ - counter up to $q$ $r$ - removed input channel number

$\mathrm{r} !$ - all input channels up to $r$

Recursive algorithm defined in Figure 2 is used to compute:

- conditioned single-sided auto and cross spectral density functions (e.g. $G_{23}$ );

- $\quad$ individual transfer functions between uncorrelated (conditioned) inputs and the output $\left(L_{i y}\right)$;

- $\quad$ individual transfer functions between (usually correlated) original inputs and the output $\left(H_{i y}\right)$;

- ordinary and partial coherence functions ( $\operatorname{coh}_{i y: r !}$ ),

- multiple coherence functions ( $\left.\operatorname{coh}_{y i}\right)$.

Based on the recursive operations shown in Figure 2, arithmetic to formulate the MISO system can be performed in the following order (in the current study $q=3$ ):

Conditioned PSDs and CSDs of relative to $2^{\text {nd }}, 3^{\text {rd }}$ until $q^{\text {th }}$ mathematical input

$$
\begin{array}{ll}
L_{r j}=G_{r j .(r-1) !} / G_{r r .(r-1) !} & r=1, \ldots,(j-1) ; j=1, \ldots, q \\
G_{i j . r !}=G_{i j .(r-1) !}-L_{r j} G_{i r .(r-1) !} & i>r, j>r, i \neq j \\
G_{j j . r !}=G_{j j .(r-1) !}-\left|L_{r j}\right|^{2} G_{r r .(r-1) !} & j>r \\
G_{i y . r !}=G_{i y .(r-1) !}-L_{r y} G_{i r .(r-1) !} & i>r \\
L_{i y}=G_{i y .(i-1) !} / G_{i i .(i-1) !} & i=1, \ldots, q
\end{array}
$$

Partial coherence functions

$$
\begin{array}{ll}
\operatorname{coh}_{i y .(i-1) !}=\left|G_{i y .(i-1) !}\right|^{2} /\left(G_{i i .(i-1) !} G_{y y .(i-1) !}\right) & i=1, \ldots, q \\
G_{y i . r !}=G_{i y .(r-1) !}-L_{r i} G_{y r .(r-1) !} & i>r \\
G_{y y . i !}=G_{y y .(i-1) !}-\left|L_{i y}\right|^{2} G_{i i .(i-1) !} & i=1, \ldots, q
\end{array}
$$

Multiple coherence function with q inputs $\mathrm{x}$ and outputs $\mathrm{y}$.

$$
\operatorname{coh}_{y: q !}=1-\left(G_{y y \cdot q !} / G_{y y}\right)=1-\left[\left(1-\operatorname{coh}_{1 y}\right)\left(1-\operatorname{coh}_{2 y .1}\right) \ldots\left(1-\operatorname{coh}_{q y \cdot(q-1) !}\right)\right]
$$

Individual transfer functions of original inputs calculated from relations of conditioned inputs

$$
H_{q y}=L_{q y} \quad H_{i y}=L_{i y}-\sum_{j=i+1}^{q} L_{i j} H_{j y} \quad i=(q-1),(q-2), \ldots, 1
$$




\section{References}

[1] Y. Huang, M.J. Griffin, Nonlinear dual-axis biodynamic response of the supine human body during longitudinal horizontal whole-body vibration, Journal of Sound and Vibration 312 (1-2) (2008) 273-295.

[2] Y. Huang, M.J. Griffin, Nonlinearity in apparent mass and transmissibility of the supine human body during vertical whole-body vibration, Journal of Sound and Vibration 324 (1-2) (2009) 429-452.

[3] N. Nawayseh, M.J. Griffin, Non-linear dual-axis biodynamic response to vertical whole-body vibration, Journal of Sound and Vibration 268 (2003) 503-523.

[4] N. Nawayseh, M.J. Griffin, Non-linear dual-axis biodynamic response to fore-and-aft whole-body vibration, Journal of Sound and Vibration 282 (2005) 831-862.

[5] P. Holmlund, R. Lundstrom, Mechanical impedance of the sitting human body in single-axis compared to multi-axis whole-body vibration exposure, Clinical Biomechanics 16 (S1) (2001) S101S110.

[6] Y. Huang, M.J. Griffin, Nonlinear dual-axis biodynamic response of the supine human body during vertical whole-body vibration, Journal of Sound and Vibration 312 (1-2) (2008) 296-315.

[7] N.J. Mansfield, S. Maeda, Comparison of the apparent masses and cross-axis apparent masses of seated humans exposed to single- and dual-axis whole-body vibration, Journal of Sound and Vibration 298 (2006) 841-853.

[8] J.S. Bendat, P.A. Palo, R.N. Coppolino, A general identification technique for nonlinear differential equations of motion, Probabilistic Engineering Mechanics 7 (1992) 43-61.

[9] J.S. Bendat, A.G. Piersol, Engineering Applications of Correlation and Spectral Analysis, second ed., John Wiley \& Sons, 1993.

[10] J.S. Bendat, A.G. Piersol, Random Data: Analysis and Measurement Procedure, fourth ed., John Wiley \& Sons, 2010.

[11] K.A. Sweitzer, Random vibration response statistics for fatigue analysis of nonlinear structures, $\mathrm{PhD}$ thesis (2006), University of Southampton, Southampton, UK.

[12] Y. Qiu, M.J. Griffin, Transmission of vibration to the backrest of a car seat evaluated with multiinput models, Journal of Sound and Vibration 274 (2004) 297-321.

[13] Y. Huang, Force harmonic distortion in the supine human body during vertical and longitudinal horizontal sinusoidal whole-body vibration, The $42^{\text {nd }}$ United Kingdom Group Meeting on Human Responses to Vibration (17-19 September 2007), Institute of Sound and Vibration Research, University of Southampton, Southampton, England.

[14] S. Mandapuram, S. Rakheja, P. Marcotte, P.E. Boileau, Analyses of biodynamic responses of seated occupants to uncorrelated fore-aft and vertical whole-body vibration, Journal of Sound and Vibration 330 (2011) 4064-4079.

[15] G.T. Rocklin, J. Crowley, H. Vold, A comparison of $H_{1}, H_{2}$, and $H_{v}$ frequency response functions, 
Proceedings of the Third International Modal Analysis Conference, Orlando, FL, vol 1 (1985) 272278.

[16] S. Siegel, N.J. Castellan, Nonparametric statistics for the behavioral sciences, New York, McGraw-Hill, 1988.

[17] G. Zheng, Y. Qiu, M.J. Griffin, An analytic model of the in-line and cross-axis apparent mass of the seated human body exposed to vertical vibration with and without a backrest, Journal of Sound and Vibration 330 (2011) 6509-6525. 